01

\title{
Параметры контура линий водяного пара при уширении гелием
}

\author{
() Н.Н. Лаврентьева, А.С. Дударёнок \\ Институт оптики атмосферы им. В.Е. Зуева Сибирского отделения РАН (ИОА СО РАН), \\ 634021 Томск, Россия \\ e-mail: Inn@iao.ru, dudaryon@iao.ru
}

Поступила в редакцию 25.05.2021 г.

В окончательной редакции 25.05.2021 г.

Принята к публикации 09.07.2021 г.

Метод средних частот применен для расчета столкновительного уширения колебательно-вращательных линий молекулы воды давлением гелия. Вычисления выполнены для широкого диапазона вращательных квантовых чисел ( $J$ от 0 до $20-$ расчет методом средних частот, $J$ от 20 до 50 - интерполяция зависимости от $J$ ), спектральный диапазон от 500 до $10000 \mathrm{~cm}^{-1}$. Полученные коэффициенты уширения линий $\mathrm{H}_{2} \mathrm{O}-\mathrm{He}$ сравниваются с литературными данными, отмечено хорошее согласие. Вычислены коэффициенты температурной зависимости полуширин линий.

Ключевые слова: уширение линий, межмолекулярные взаимодействия, метод средних частот, температурный показатель.

DOI: $10.21883 / \mathrm{OS} .2021 .10 .51488 .2334-21$

Представленные данные требуются, в первую очередь, для астрофизических приложений, в частности, при моделировании спектров низкотемпературных звезд, где необходимо знание ударных параметров контура линий $\mathrm{H}_{2} \mathrm{O}$ при соударениях с гелием и водородом в широком интервале температур. Водород и гелий являются самыми распространенными веществами во Вселенной, из них состоит большая часть атмосфер холодных звезд, планет-гигантов и экзопланет (планет вне Солнечной системы). Уширение колебательно-вращательных линий водяного пара давлением водорода и гелия при высоких температурах изучено слабо. В данной работе исследуются уширение колебательно-вращательных линий молекулы воды давлением гелия при высоких температурах, вплоть до $2000 \mathrm{~K}$.

Имеющихся в литературе данных явно недостаточно: в [1-16] представлены одиночные измерения коэффициентов уширения $\mathrm{H}_{2} \mathrm{O}-\mathrm{He}$, наиболее обширные экспериментальные значения приведены в [17-23]. Расчетные данные представлены в [23] вместе с измеренными коэффициентами уширения линий водяного пара давлением гелия для обширного списка линий из 11 полос поглощения. Коэффициенты температурной зависимости в литературе получены только для случая уширения давлением гелия, так же как и давлением водорода, двух линий $\left(3_{13} \leftarrow 22_{20}\right.$ и $\left.4_{14} \leftarrow 3_{21}\right)$ при температурах 80-600 K [1-3]. В работе [12] проведены низкотемпературные измерения для линии $1_{10} \leftarrow 1_{01}$ в диапазоне $17-200 \mathrm{~K}$, для данной линии найдена очень слабая температурная зависимость.

Для расчета ударных параметров контура линий в настоящее время используется несколько методов, к числу которых относятся различные модификации метода Робера-Бонами [24], модифицированный комплексный формализм Робера-Бонами [25], полуэмпирический ме- тод [26]. В последнее время предложен метод средних частот [27], который позволяет быстро рассчитывать коэффициенты уширения колебательно-вращательных линий молекул типа асимметричного волчка на основе небольшого количества эмпирических данных. Идея метода заключается в том, что из всей расчетной схемы выделена часть величин, которые отражают влияние буферной молекулы на внутреннее состояние поглощающей молекулы. Эти величины сильно зависят от квантовых индексов поглощающей молекулы. Из них компонуется величина, которая названа средней частотой столкновительных переходов. Под виртуальными переходами понимаем все возможные переходы с определенного энергетического уровня, обусловленные столкновениями. Средняя частота виртуальных переходов для линии $i \rightarrow f$ определяется следующим выражением:

$$
\begin{aligned}
\bar{\omega}_{i f}= & \frac{1}{W}\left\{\sum_{i^{\prime}}\left(2 i^{\prime}+1\right) D^{2}\left(i i^{\prime} \mid l\right) f\left(\omega_{i i^{\prime}}\right) \omega_{i i^{\prime}}\right. \\
& \left.+\sum_{f^{\prime}}\left(2 f^{\prime}+1\right) D^{2}\left(f f^{\prime} \mid l\right) f\left(\omega_{f f^{\prime}}\right) \omega_{f f^{\prime}}\right\},
\end{aligned}
$$

где

$$
\begin{aligned}
W= & \sum_{i^{\prime}}\left(2 i^{\prime}+1\right) D^{2}\left(i i^{\prime} \mid l\right) f\left(\omega_{i i^{\prime}}\right) \\
& +\sum_{f^{\prime}}\left(2 f^{\prime}+1\right) D^{2}\left(f f^{\prime} \mid l\right) f\left(\omega_{f f^{\prime}}\right) .
\end{aligned}
$$

Здесь в формулу включены частоты столкновительных (виртуальных) переходов $\omega_{i i^{\prime}}, \omega_{f f^{\prime}}$, матричные элементы дипольного $(l=1)$, квадрупольного $(l=2)$ моментов виртуальных переходов $D^{2}\left(i i^{\prime} \mid l\right), D^{2}\left(f f^{\prime} \mid l\right)$ и резонансные функции $f\left(\omega_{i i^{\prime}}\right), f\left(\omega_{f f^{\prime}}\right)$. Видно, что усреднение проходит по всем виртуальным переходам, а также 


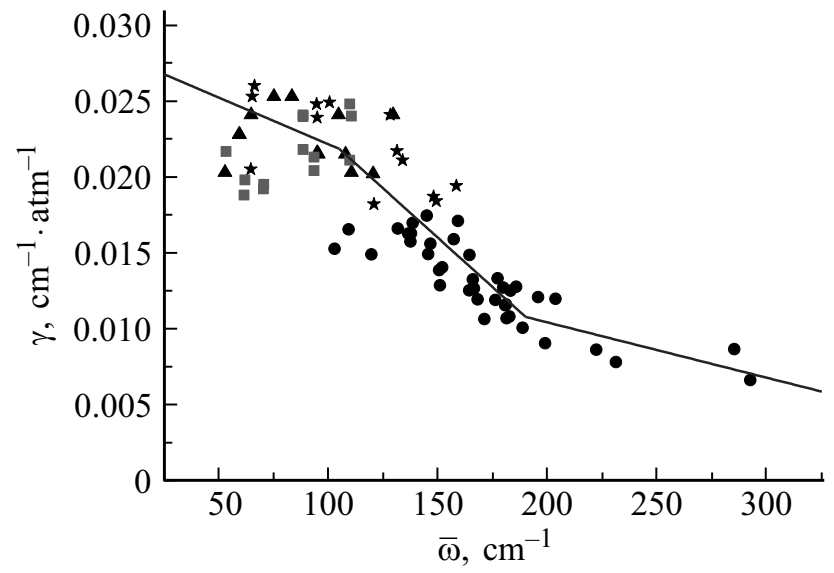

Рис. 1. Экспериментальные полуширины линий $\mathrm{H}_{2} \mathrm{O}-\mathrm{He}$ в зависимости от средней частоты столкновительных переходов. Квадраты - данные из [3-6,9,10,12], треугольники [20], кружки [21], звездочки [22], сплошная линия - аппроксимация.

по нижнему и верхнему энергетическим состояниям, образующим данный переход $i \rightarrow f$.

Зная соответствие между величинами $\bar{\omega}_{i f}$ (рассчитаны заранее для всех возможных переходов до $J=20)$ и квантовой идентификацией линий, сопоставляем столкновительные коэффициенты уширения линий со значениями $\bar{\omega}_{i f}$. По последней зависимости получаем аппроксимацию в виде некоторого простого выражения. Обычно это прямолинейная или квадратичная зависимость, по которой возможно восстановить полуширины линий.

При применении метода средних частот прежде всего необходимо выявить верифицированные литературные данные. Поскольку экспериментальные полуширины линий водяного пара, индуцированные давлением гелия, из работ [20-22] хорошо согласуются между собой, именно их мы использовали для реализации метода средних частот. На рис. 1 представлена зависимость коэффициентов уширения от средней частоты столкновительных переходов для случая $\mathrm{H}_{2} \mathrm{O}-\mathrm{He}$, а также соответствующая аппроксимация в зависимости от средней частоты столкновительных переходов. Здесь наиболее обширные измерения [20-22] показаны символами черного цвета, все одиночные данные [3-6,9,10,12] - серыми квадратами.

Получена следующая аппроксимация тремя отрезками прямой:

$$
\begin{aligned}
& \gamma_{i f}=-0.0000615 \bar{\omega}_{i f}+0.02830 \text { при } 0<\bar{\omega}_{i f} \leq 105, \\
& \gamma_{i f}=-0.0001302 \bar{\omega}_{i f}+0.03552 \text { при } 105<\bar{\omega}_{i f} \leq 190, \\
& \gamma_{i f}=-0.0000364 \bar{\omega}_{i f}+0.01769 \text { при } \bar{\omega}_{i f}>190 .
\end{aligned}
$$

Затем коэффициенты уширения линий были рассчитаны по формуле (2) для значений вращательного квантового числа $J \leq 20$, часть полученных таким образом данных представлена в [28]. Для более высо- ких значений $20<J \leq 50$ экспериментальных данных нет, поэтому для их получения была использована аппроксимация величин $\gamma$ от $J$. Для этой цели были собраны выверенные данные для нижних колебательных полос [3-5,9,10,12,20-22], значения коэффициентов уширения $\mathrm{H}_{2} \mathrm{O}-\mathrm{He}$ были построены в зависимости от вращательного квантового числа $J$ и затем усреднены по квантовым числам $K_{a}, K_{c}$. Измеренные коэффициенты уширения линий вращательной полосы, полос $v_{2}$ и $2 v_{1}$ в зависимости от $J$ приведены на рис. 2. Также на рисунке нанесены усредненные значения полуширин линий по всем квантовым числам $K_{a}$ и аппроксимация.

Вращательная зависимость полуширин линий для уширения гелием в сравнении с уширением водородом [29], построенная по литературным данным, показана на рис. 3. Видно, что полуширины линий $\mathrm{H}_{2} \mathrm{O}-\mathrm{H}_{2}$ больше, чем полуширины линий $\mathrm{H}_{2} \mathrm{O}-\mathrm{He}$ в среднем в 2.5 раза, что обусловлено наличием квадрупольного момента у молекулы $\mathrm{H}_{2}$.

Вычисленные с помощью средних частот, насчитанных для вращательной полосы, полуширины линий для системы $\mathrm{H}_{2} \mathrm{O}-\mathrm{H}_{2}$ как в чисто вращательной полосе, так и в других колебательных полосах поглощения хорошо согласуются с экспериментальными данными благодаря слабой колебательной зависимости полуширин линий. Однако, как показано в работе [23], измеренные полуширины линий для смеси газов $\mathrm{H}_{2} \mathrm{O}-\mathrm{He}$ могут меняться более чем на $10 \%$ при переходе от одной полосы поглощения к другой. Поэтому для такого буферного газа как гелий для получения более точных полуширин линий средние частоты были изменены для возбужденных состояний. На рис. 4 показано сравнение

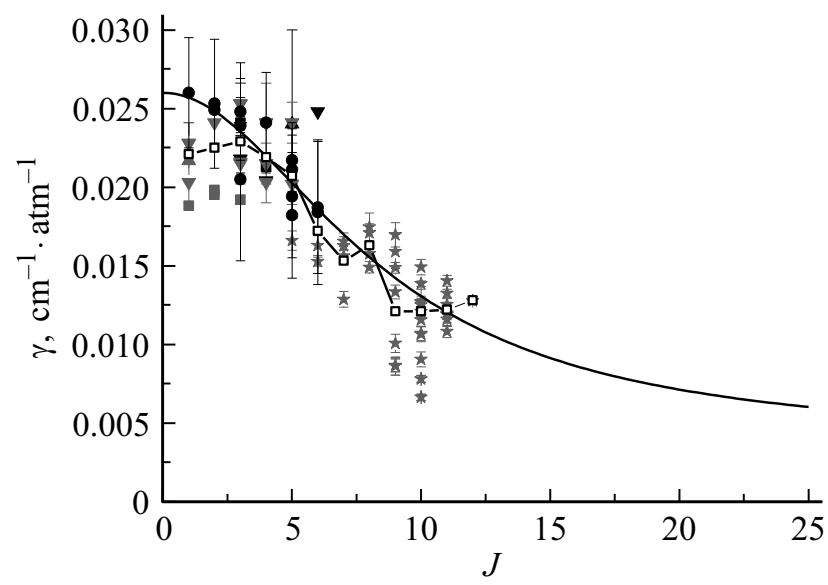

Рис. 2. Вращательная зависимость полуширин линий для случая $\mathrm{H}_{2} \mathrm{O}-\mathrm{He}$. Черными квадратами, треугольниками, перевернутыми треугольниками, звездочками обозначены данные [3], [4], [5], [9] соответственно; серыми квадратами, треугольниками, перевернутыми треугольниками, звездами $[10],[12],[20],[21]$ соответственно; черными кружками [22]; линией со светлыми квадратами - средние значения для определенного значения вращательного квантового числа $J$; сплошная линия — аппроксимация. 


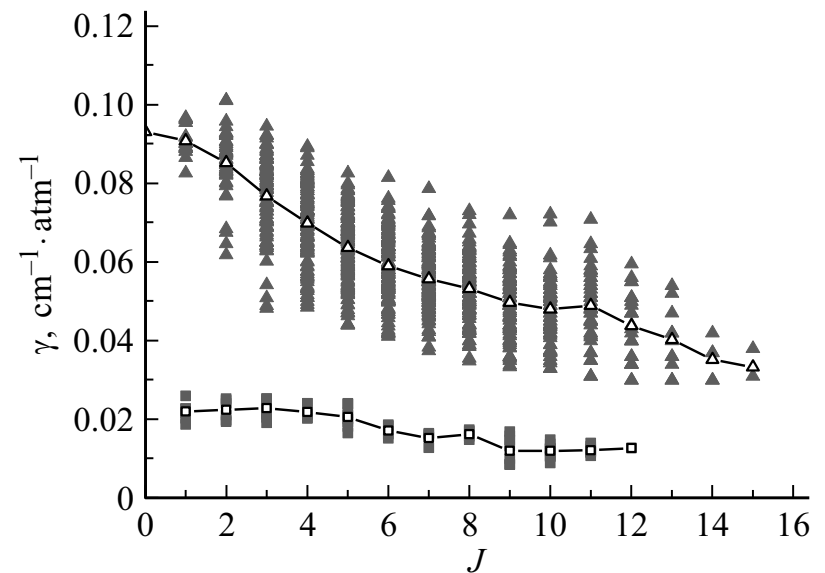

Рис. 3. Коэффициенты уширения линий водяного пара давлением водорода и гелия в зависимости от вращательного квантового числа $J$. Серыми треугольниками и квадратами обозначены наборы измеренных величин, линией со светлыми треугольниками и квадратами - средние значения для определенного значения вращательного квантового числа $J$ для смесей $\mathrm{H}_{2} \mathrm{O}-\mathrm{H}_{2}$ и $\mathrm{H}_{2} \mathrm{O}-\mathrm{He}$ соответственно.

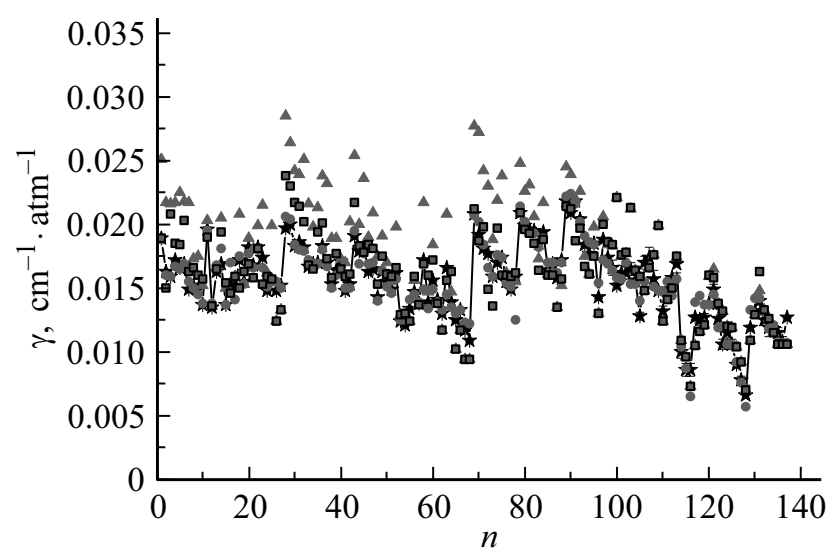

Рис. 4. Вычисленные по методу средних частот без учета формулы (4) (треугольники) и с применением формулы (4) (квадраты) коэффициенты уширения линий $\mathrm{H}_{2} \mathrm{O}$ давлением $\mathrm{He}$ в сравнении с экспериментальными (линия со звездочками) и теоретическими (кружки) данными [23] Номер линии $n$ соответствует увеличению частоты перехода.

рассчитанных нами полуширин линий без учета колебательной зависимости с полученными в [23] экспериментальными и вычисленными данными. Максимальное рассогласование в данных наблюдается для небольших частот столкновительных переходов.

В работе [27] для уточнения колебательной зависимости полуширин линий для системы $\mathrm{H}_{2} \mathrm{O}-\mathrm{N}_{2}$ была предложена формула для расчета средних частот столкновительных переходов в колебательной полосе $\bar{\omega}\left(v_{2} v_{2} v_{3}\right)$ как суммы средней частоты вращательной полосы и разностей средних частот для первого возбужденного состояния $v_{1}, v_{2}, v_{3}$, умноженных на количество колебательных квантов, соответствующих определенно- му колебанию:

$$
\begin{aligned}
& \bar{\omega}\left(v_{2} v_{2} v_{3}\right)=\bar{\omega}(000)+v_{1}[\bar{\omega}(000)-\bar{\omega}(000)] \\
& \quad+v_{2}[\bar{\omega}(010)-\bar{\omega}(000)]+v_{3}[\bar{\omega}(001)-\bar{\omega}(000)] .
\end{aligned}
$$

Формула адекватно описывает поведение уширения линий со слабой зависимостью от колебательных квантовых чисел (например, в случае $\mathrm{H}_{2} \mathrm{O}-\mathrm{N}_{2}$ ). Однако в случае уширения атомарным гелием, когда проявляется сильная колебательная зависимость полуширин, ее использование неприемлемо. Поэтому указанная формула была модифицирована введением множителей перед разностями, которые были получены из экспериментальных полуширин линий в полосах $2 v_{1}, 2 v_{2}, 2 v_{2}+v_{3}$ [23] (использовалось по одному переходу в каждой полосе):

$$
\begin{aligned}
& \bar{\omega}\left(v_{2} v_{2} v_{3}\right)=\bar{\omega}(000)+a_{1} v_{1}[\bar{\omega}(000)-\bar{\omega}(000)] \\
& \quad+a_{2} v_{2}[\bar{\omega}(010)-\bar{\omega}(000)]+a_{3} v_{3}[\bar{\omega}(001)-\bar{\omega}(000)]
\end{aligned}
$$

где

$$
\begin{aligned}
& a_{1}=0.14954 \bar{\omega}-22.431, \\
& a_{1}=-0.06477 \bar{\omega}+9.715, \\
& a_{3}=0.08684 \bar{\omega}-14.527 .
\end{aligned}
$$

На рис. 5 представлена зависимость экспериментальных полуширин линий [3-6,9,10,12,20-23] от средней частоты столкновительных переходов, полученной по формуле (4). Измеренные значения [23] изображены отдельно для каждой колебательной полосы. Видно, что после пересчета параметров $\bar{\omega}$ по формуле (4) коэффициенты аппроксимации (2), полученные для низких

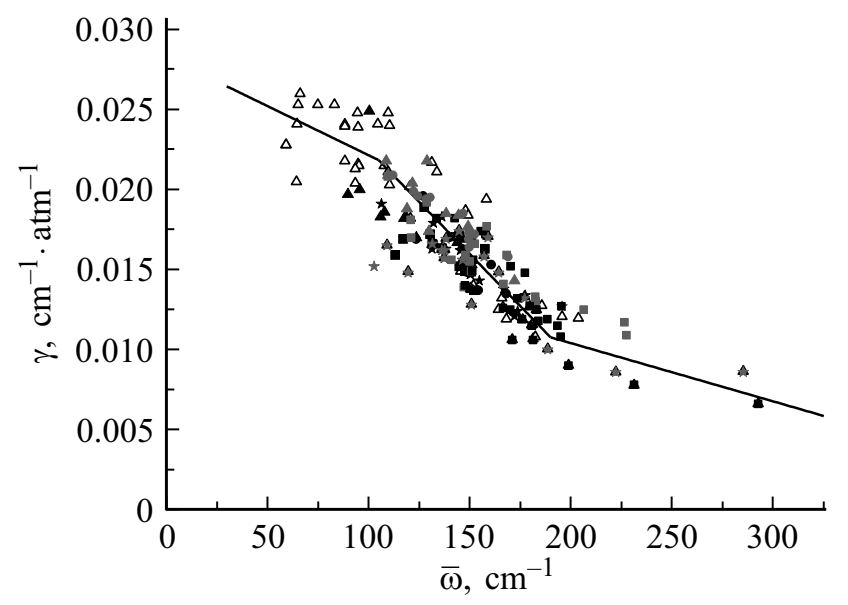

Рис. 5. Коэффициенты уширения линий $\mathrm{H}_{2} \mathrm{O}$ давлением Не в зависимости от частот столкновительных переходов. Черными квадратами, кружками, треугольниками, звездочками обозначены измеренные величины [23] в полосах $2 v_{2}, v_{1}, v_{1}+v_{2}, v_{2}+v_{3}$ соответственно; серыми квадратами, кружками, треугольниками, звездочками обозначены измеренные величины [23] в полосах $2 v_{2}+v_{3}, 2 v_{1}, v_{1}+v_{3}$, во вращательной полосе соответственно; светлые треугольники - данные [3-6,9,10,12,20-22]; сплошная линия - аппроксимация для метода средних частот. 
колебательных полос, будут прежние. Среднеквадратичные отклонения рассчитанных полуширин линий без использования модификации (4) и с использованием модификации (4) от экспериментальных данных [23] будут равны 0.0029 и $0.0017 \mathrm{~cm}^{-1} \cdot \mathrm{atm}^{-1}$ соответственно.

Для приложений в астрофизике, в частности, при моделировании спектров низкотемпературных звезд необходимо знание ударных параметров контуров линий в широком интервале температур. Коэффициенты температурной зависимости $N$ для уширения двух линий водяного пара $\left(3_{13} \leftarrow 22_{20}\right.$ и $\left.4_{14} \leftarrow 3_{21}\right)$ давлением гелия, определенные в интервале температур 80-600 K, приведены в [1-3]. В данной работе температурный показатель для случая $\mathrm{H}_{2} \mathrm{O}-\mathrm{He}$ был определен как отношение к величинам $N$ для случая $\mathrm{H}_{2} \mathrm{O}-\mathrm{H}_{2}$ :

$$
N^{\mathrm{H}_{2} \mathrm{O}-\mathrm{He}}=0.576 N^{\mathrm{H}_{2} \mathrm{O}-\mathrm{H}_{2}},
$$

где $N^{\mathrm{H}_{2} \mathrm{O}-\mathrm{H}_{2}}$ получен по методу средних частот в [29]. Множитель в соотношении (5) был получен из измеренных данных [3].

\section{Заключение}

В работе рассчитаны коэффициенты уширения линий и их температурные показатели для смеси $\mathrm{H}_{2} \mathrm{O}-\mathrm{He}$. Квантовое число полного углового момента варьируется в интервале от 0 до 50. Показано, что при исследовании уширения линий водяного пара давлением гелия в широком спектральном диапазоне необходимо учитывать зависимость от колебательного возбуждения. Получено аппроксимационное выражение для колебательной зависимости, которое позволяет адекватно описать имеющиеся экспериментальные данные. Таким образом, разработанный авторами метод средних частот, основанный на оценке вкладов столкновительных переходов в полуширину спектральной линии, дает возможность рассчитывать полуширины линий молекул типа асимметричного волчка с точностью, близкой к точности современных расчетных и экспериментальных методов, не прибегая к сложной вычислительной схеме. Значения полуширин линий $\mathrm{H}_{2} \mathrm{O}-\mathrm{He}$, вычисленные с помощью предлагаемого метода, хорошо согласуются с различными экспериментальными и расчетными данными.

\section{Финансирование работы}

Работа выполнена в рамках государственного задания ИОА СО РАН и гранта РФФИ № 19-03-00389.

\section{Конфликт интересов}

Авторы заявляют, что у них нет конфликта интересов.

\section{Список литературы}

[1] Godon M., Bauer A. // Chem. Phys. Lett. 1988. V. 147. N 2-3. P. 189-191. doi 10.1016/0009-2614(88)85081-4

[2] Goyette T.M., Lucia F.C. // J. Mol. Spectrosc. 1990. V. 143. N 2. P. 346-358. doi 10.1016/0022-2852(91)90099-V

[3] Dutta J.M., Jones C.R., Goyette T.M., Lucia F.C. // Icarus. 1993. V. 102. N 2. P. 232-239. doi 10.1006/icar.1993.1046

[4] Lazarev V.V., Ponomarev Y.N., Sumpf B., Fleischmann O., Waschull J., Kronfeldt H.D., Stroinova V.N. // J. Mol. Spectrosc. 1995. V. 173. N 1. P. 177-193. doi 10.1006/jmsp.1995.1227

[5] Gamache R.R., Lynch R., Brown L.R. // JQSRT. 1996. V. 56. N 4. P. 471-487. doi 10.1016/0022-4073(96)00098-2

[6] Lepere M., Henry A., Valentin A., Camy-Peyret C. // J. Mol. Spectrosc. 2001. V. 208. N 1. P. 25-31. doi 10.1006/jmsp.2001.8372

[7] Zeninary V., Parvitte B., Courtois D., Pouchetm I., Durry G., Ponomarev Yu.N. // Atm. Ocean. Opt. 2003. V. 16. N 3. P. 189-192.

[8] Zeninary V., Parvitte B., Courtois D., Lavrentieva N.N., Ponomarev Yu.N., Durry G. // Mol. Phys. 2004. V. 102. N 1617. P. $1697-1706$. doi 10.1080/00268970412331287133

[9] Golubiatnikov G.Yu. // J. Mol. Spectrosc. 2005. V. 230. N 2. P. 196-198. doi 10.1016/j.jms.2004.10.011

[10] Brown L.R., Benner D.C., Devi V.M., Smith M.A.H., Toth R.A. // J. Mol. Structure. 2005. V. 742. N 1-3. P. 111-122. doi 10.1016/j.molstruc.2004.12.059

[11] Lisak D., Hodges J.T., Ciuryło R. // Phys. Rev. A. 2006. V. 73. P. 012507.

[12] Dick M.J., Drouin B.J., Pearson J.C. // JQSRT. 2009. V. 110. N 9-10. P. 619-627. doi 10.1016/j.jqsrt.2008.11.012

[13] Solodov A.M., Starikov V.I. // Mol. Phys. 2009. V. 107. N 1. P. 43-51. doi 10.1080/00268970802698655

[14] Osipov K.Yu., Kapitanov V.A., Protasevich A.E., Pereslavtseva A.A., Ponurovsky Ya.Ya. // JQSRT. 2014. V. 142. P. 1-8. doi 10.1016/j.jqsrt.2014.03.003

[15] Starikov V.I. // Opt. Spectrosc. 2014. V. 116. N 2. P. 238-247.

[16] Claveau C., Henry A., Hurtmans D., Valentin A. // JQSRT. 2001. V. 68. N 3. P. 273-298. doi 10.1016/S00224073(00)00025-X

[17] Protasevich A.E., Starikov V.I. // Opt. Spectrosc. 2005. V. 98. N 4. P. $577-584$.

[18] Solodov A.M., Starikov V.I. // Opt. Spectrosc. 2008. V. 108. N 1. P. 19-26.

[19] Claveau C., Valentin A. // Mol. Phys. 2009. V. 107. N 14. P. 1417-1422. doi 10.1080/00268970902911404

[20] Lucchesinia A., Gozzini S., Gabbanini C. // Eur. Phys. J. D. 2000. V. 8. P. 223-226.

[21] Steyert D.W., Wang W.F., Sirota J.M., Donahue N.M., Reuter D.C. // JQSRT. 2004. V. 83. N 2. P. 183-191. doi 10.1016/S0022-4073(02)00300-X

[22] Poddar P., Mitra S., Hossain M.M., Biswas D., Ghosh P.N., Ray B. // Mol. Phys. 2010. V. 108. N 15. P. 1957-1964. doi 10.1080/00268976.2010.499376

[23] Petrova T.M., Solodov A.M., Solodov A.A., Starikov V.I. // JQSRT. 2013. V. 129. P. 241-253. doi 10.1016/j.jqsrt.2013.06.021

[24] Robert D., Bonamy J. // J. Phys. France. 1979. V. 40. N 10. P. 923-943. doi 10.1051/jphys:019790040010092300

[25] Ma Q., Tipping R.H., Boulet C. // JQSRT. 2007. V. 103. P. 588-96. doi 10.1016/j.jqsrt.2006.08.001 
[26] Bykov A., Lavrentieva N., Sinitsa L. // Mol. Phys. 2004. V. 102. N 14-15. P. 1653-1658. doi $10.1080 / 00268970410001725765$

[27] Дударёнок А.С., Лаврентьева Н.Н., Ма К. // Оптика атмосферы и океана. 2015. Т. 28. № 8. С. 675681; Dudaryonok A.S., Lavrentieva N.N., Ma Q. // Atm. Ocean. Opt. 2015. V. 28. N 6. P. 503-509. doi 10.1134/S1024856015060056

[28] Barton E.J., Hill C., Yurchenko Sergei N., Tennyson J., Dudaryonok A.S., Lavrentieva N.N. // JQSRT. 2017. V. 187. P. 453-460. doi 10.1016/j.jqsrt.2016.10.024

[29] Лаврентьева Н.Н., Дударёнок А.С. // Оптика атмосферы и океана. 2014. Т. 29. № 10. С. 828-832. 